Article

\title{
Vascular Access Flow during Dialysis: Does Needle Orientation Matter?
}

\author{
Georgios Tsangalis * and Valérie Loizon
}

check for updates

Citation: Tsangalis, G.; Loizon, V. Vascular Access Flow during Dialysis: Does Needle Orientation Matter? Kidney Dial. 2021, 1, 138-146. https:// doi.org/10.3390/kidneydial1020017

Academic Editor: Giorgina Barbara Piccoli

Received: 21 October 2021

Accepted: 22 November 2021

Published: 30 November 2021

Publisher's Note: MDPI stays neutral with regard to jurisdictional claims in published maps and institutional affiliations.

Copyright: (c) 2021 by the authors. Licensee MDPI, Basel, Switzerland. This article is an open access article distributed under the terms and conditions of the Creative Commons Attribution (CC BY) license (https:// creativecommons.org/licenses/by/ $4.0 /)$.
Centre de Néphrologie de Montargis, 658 Bis, Rue de Bourgoins, 45200 Amilly, France; valerie.loizon@wanadoo.fr * Correspondence: gtsangalis4@gmail.com; Tel.: +33-667047180

\begin{abstract}
Background: Monitoring of vascular access outflow (VAO) in dialysis is based on the indicator dilution method by ultrasound (UD). The role of arterial needle orientation in VAO measurement is not clear. We compared the impact of the retrograde (RET) versus the antegrade orientation (ANT) in terms of (a) VAO (UD) and (b) dialysis adequacy. Moreover, we compared VAO (UD ANT and RET orientation) with VAO measured by Doppler ultrasound. Methods: 22 patients participated in the study. Inclusion criteria: Dialysis $>6$ months with a functioning AVF, no stenosis, no active infection, EF $>45 \%$ and informed consent. 4 flow measurements were taken on the same dialysis day (4 consecutive weeks). To account for blood pressure variation, we "corrected" VAO for a mean arterial pressure of $100 \mathrm{mmHg}$. Doppler VAO was measured just before dialysis. Means were compared by the paired $t$-test. For correlation and agreement, linear regression and Bland-Altman analysis were performed respectively. Results: Mean VAO (UD) was higher in the (ANT) versus the (RET) orientation: $1286.17 \mathrm{~mL} / \mathrm{min}(\mathrm{SD}=455.78,95 \% \mathrm{CI}=1084-1488)$ versus $1189.96 \mathrm{~mL} / \mathrm{min}(\mathrm{SD}=401.05$, $95 \% \mathrm{CI}=1012-1368)(p=0.013)$ with a mean difference of $96.21 \mathrm{~mL} / \mathrm{min}(5.66 \%)$. Mean Kt/V (RET orientation) was $1.57(\mathrm{SD}=0.10,95 \% \mathrm{CI}=1.52-1.61)$ versus $1,55(\mathrm{SD}=0.10,95 \% \mathrm{CI}=1.50-1.60)(\mathrm{ANT})$ orientation $(p=0.062)$. Recirculation was always $0 \%$. The mean VAO (Doppler) was $1079.54 \mathrm{~mL} / \mathrm{min}$ $(\mathrm{SD}=356.04,95 \% \mathrm{CI}=922-1237), 16 \%$ lower than VAO measured by UD with (ANT) orientation $(p=0.009)$ and $9.3 \%$ lower than the VAO in the (RET) orientation $(p=0.113)$. Linear regression analysis showed that VA flows (ANT versus RET) orientation of the needle correlates well between them $(r=0.93, p<0.001)$ but show poor agreement (Bland-Altman analysis). Conclusion: VAO (UD) in the RET orientation was significantly lower than VAO in the ANT orientation and more consistent with VAO assessed by Doppler without influencing dialysis adequacy. Therefore, when using UD for VAO surveillance, the RET orientation should be used.
\end{abstract}

Keywords: hemodialysis; AV fistula; ultrasonography-Doppler evaluation; techniques and procedures; prosthetic graft; new devices

\section{Introduction}

Almost a century after the first dialysis treatment in humans (1924 by George Haas) [1] and 55 years after the first autogenous radio cephalic fistula (Brescia-Appel-Cimino in 1966) [2], vascular access still remains the Achilles' heel of modern hemodialysis treatment.

Cannulation practices differ significantly between countries and dialysis centers as well. Site selection (area, rope ladder, and buttonhole), needle size, and arterial needle orientation remain subjects of controversy. The venous needle orientation is not a subject of debate since the venous needle returns blood to the body and should always point to the direction of blood flow (antegrade). Concerning the arterial needle orientation, two options exist: (a) antegrade (direction of blood flow) and (b) retrograde (in the opposite direction). Parisotto et al. [3] showed that area cannulation versus rope-ladder or buttonhole and the retrograde direction of the arterial needle with bevel down versus antegrade orientation with bevel up increased the risk of access failure. It is important to underline that the same authors clearly stated that concerning needle orientation, all other options (antegrade with 
the bevel down and retrograde with the bevel up) were not associated with a hazard ratio significantly different from 1.00 .

The effect of the retrograde placement of the arterial needle on dialysis adequacy is a matter of debate. According to Gauly et al. [4], the retrograde placement of the arterial needle showed a higher odds ratio to attain a Kt/ $\mathrm{V}$ of 1.2 versus the antegrade orientation. Reyes et al. [5] conducted a randomized controlled trial in a non-diabetic dialysis population with no severe heart issues, showing that adequacy was higher with antegrade versus retrograde cannulation although both needle directions met the KDOQI guidelines. Ozmen et al. [6] studied the same issue in 22 chronic hemodialysis patients for more than 6 months. Contrary to the findings of Gauly et al., the two cannulation strategies (antegrade versus retrograde) resulted in the same urea reduction ratio and $\mathrm{eKt} / \mathrm{V}(74.2+/-7.2 \%)$ and $1.57+/-0.33$, respectively.

The hemodynamic consequences of needle orientation and rotation were studied as well. Fulker et al. [7] found that needle rotation had no hemodynamic benefit. In the same study, the retrograde orientation of the arterial needle resulted in a smooth flow in the area close to the needle and a small region of oscillatory shear whereas the antegrade orientation produced a large region of oscillatory shear. Moreover, flow through the arterial back eye was found to be more efficient in the retrograde orientation.

The National Kidney Foundation Kidney Disease Outcomes Quality Initiative (NKFKDOQI) guidelines recommend that concerning native arteriovenous fistulae (AVF) clinical monitoring is primary, while surveillance findings are supplementary [8]. Surveillance of VAO is common practice in our dialysis unit and combined with clinical monitoring increases the detection of clinically significant stenosis.

The velocity of ultrasound in the blood is determined primarily by its blood protein concentration. The UD technique uses Flow/dilution Sensors measuring ultrasound velocity. A bolus of isotonic saline is introduced into the bloodstream, mixes with blood, and reduces the ultrasound velocity. Sensors positioned in the arterial and venous blood lines register an indicator dilution curve. The bloodline connections to the needles are reversed so that the arterial inlet removing blood is downstream from the venous outlet and the venous outlet faces the access flow stream thus creating a good mixing zone upstream from the venous outlet.

In his original article [9], Krivitski clearly stated that "To use the dilution method for access blood flow measurement during hemodialysis, the bloodline connection to the needles must be reversed so that the arterial inlet removing blood is now downstream from the venous outlet and the venous outlet faces the access flow stream. This creates a good mixing zone upstream from the venous outlet". He also emphasized that "when the venous outlet does not face the access stream (incorrect orientation) the situation becomes unpredictable".

The abovementioned suggestion of the inventor of the UD device is not respected in everyday clinical practice. For VAO measurements the usual needle orientation is the antegrade one (pointing to the direction of blood flow) for both needles.

All in all, data suggest that the retrograde orientation of the arterial needle has probable beneficial effects for vascular access, is not related to an increased risk of failure (with bevel up), and results in equal or better dialysis efficiency. Concerning the accuracy of VAO measurements, the retrograde orientation of the arterial needle favors indicator mixing and therefore should be used whenever access blood flow is measured with the UD device.

For all these reasons, we decided to compare the retrograde arterial needle orientation with the commonly used antegrade orientation in terms of (a) VAO measurements by the UD device and (b) quality of dialysis. Finally, we compared VAO measurements using both RET and ANT arterial needle orientation with VAO measured by Doppler ultrasound. 


\section{Methods}

This was a prospective single-center observational study of 22 patients treated by thrice-weekly hemodialysis for more than 6 months.

\subsection{Protocol}

135 patients are dialyzed on a thrice-weekly basis in our dialysis center. $10(7.4 \%)$ from a central venous catheter (CVC), 2 (1.48\%) from an arteriovenous graft (AVG), and 123 from a fistula (91.1\%). Patients were included in the study based on the following criteria: (1) Informed consent, (2) on dialysis for more than 6 months, (3) absence of active infection, (4) absence of severe systolic heart failure $(\mathrm{EF}<45 \%)$. Based on these criteria, 101 patients were excluded: 59 patients since they did not consent (due to fear of damaging dialysis access from the retrograde orientation of the arterial needle), 9 for severe heart failure, 11 for active infection, and 22 as they were not on dialysis for more than 6 months. Finally, 22 patients started and finished the study. All of them were dialyzed through a native arteriovenous fistula (no one had earlier arteriovenous access) and had $\mathrm{EF}>50 \%$. Measurements were performed over 4 dialysis sessions for each patient, two with the arterial needle facing (RET) and two with the arterial needle not facing (ANT) the bloodstream (Supplementary Figures). Two measurements were performed on each day on each patient and the mean of these two measurements was recorded. All 22 patients were followed for 6 months (January-June 2021). The study was approved by the medical research committee of our institution.

In our practice, VAs are cannulated regularly using the antegrade orientation for the arterial needle. Needle tip distance was always $\geq 4 \mathrm{~cm}$. Both the rope ladder and the buttonhole technique are used but for this study, we only included patients cannulated (bevel up) with the rope ladder technique.

We alternated needle orientation: Retrograde-anterograde-retrograde-anterograde. Cannulation of all AVFs and flow measurements were performed by the same staff member to respect needle distancing and strengthen the internal validity of measurements. Moreover, tip position was verified (before measurements) with ultrasound to avoid needle tip misplacement. Flow measurements were performed with the HDO3 Hemodialysis Monitor (Transonic Systems, Ithaca, NY, USA) as per protocol: Blood pump set at $250 \mathrm{~mL} / \mathrm{min}$, measurements during the first 20 min of dialysis (maximal hemodynamic stability).

Measurement of vascular access flow with Doppler ultrasound was performed in the day that preceded the first flow measurement ( 3 measures-mean reported). Of the 22 patients included in the study, no one had stenosis on Doppler ultrasound.

Blood pressure directly influences $\mathrm{VAO}$ and although measurements were made during the first 20 min of the dialysis session (to maximize hemodynamic stability), the impact of blood pressure on the access outflow should be taken into consideration. To face this issue, we recorded systolic and diastolic arterial blood pressures during VAO measurements. These recordings enabled us to calculate the mean arterial pressure (MAP) that corresponded to each measurement of access outflow based on the following equation:

$$
\mathrm{MAP}=\mathrm{DBP}+(\mathrm{SBP}-\mathrm{DBP}) / 3
$$

To compare VAO and since MAP differed between measurements, we decided to "correct" VAO for a MAP of $100 \mathrm{mmHg}$ according to the following equation:

"VAO corrected for $100 \mathrm{mmHg}$ of MAP" = $100 \times \mathrm{VAO}$ (measured by UD)/MAP, with MAP calculated by Equation (1).

This "correction" allowed us to take into account the role of blood pressure in the VAO measurements. Consequently, we compared the "corrected" VAO results obtained with the arterial needle in the retrograde and antegrade orientation.

All 22 patients gave written consent to participate in the study. 


\subsection{Primary Outcomes-Definitions}

VAO-UD (RET): Vascular access output ( $\mathrm{mL} / \mathrm{min}$ ) measured by the UD technique expressed in $\mathrm{mL} / \mathrm{min}$, with the arterial needle facing the bloodstream (RETROGRADE orientation). The venous needle did not face the bloodstream. Measurements were made during the first $20 \mathrm{~min}$ of the dialysis session and results were "normalized" for MAP as previously described.

Recirculation (RET): Recirculation (expressed as \%) using the UD technique with the arterial needle facing the bloodstream (RETROGRADE orientation).

VAO-UD (ANT): Vascular access output $(\mathrm{mL} / \mathrm{min})$ measured by the UD technique expressed in $\mathrm{mL} / \mathrm{min}$, with the arterial needle not facing the bloodstream (ANTEGRADE orientation). The venous needle did not face the bloodstream. Measurements were made during the first 20 min of the dialysis session and results were "normalized" for MAP as previously described.

Recirculation (ANT): Recirculation (expressed as \%) using the UD technique with the arterial needle not facing the bloodstream (ANTEGRADE orientation).

VAO (Doppler): Vascular access output expressed in $\mathrm{mL} / \mathrm{min}$ in the day preceding the first flow measurement ( 3 measures in the brachial artery-mean reported). Of the 22 patients included in the study, no one had stenosis on Doppler ultrasound.

\subsection{Data Analysis}

Data were normally distributed and thus compared by the paired Student-t-test. A probability of $p<0.05$ was assumed to reject the null hypothesis (Null hypothesis: No difference between methods).

To test for correlation, linear regression analysis was performed. Bland and Altman analysis was used to test for agreement between VAO measured in the (ANT) and the (RET) needle orientation.

\section{Results}

All 22 patients completed the study. 12 were females (54.5\%) and 10 males (45.4\%). 20 patients had distal radio cephalic AVFs (90\%), 1 patient had a proximal radio-cephalic AVF and 1 patient had a brachiobasilic AVF. Patients' ages ranged from 51-89 years with a mean of 76.27 years $(S D=9.66)$. Table 1 provides the means of readings for:

1. VAO measured by Doppler.

2. MAP $(\mathrm{mm} \mathrm{Hg})$ during measurements in antegrade and retrograde orientation.

3. VAO in antegrade (ANT) and retrograde (RET) position after "normalization" for MAP $(100 \mathrm{mmHg})$.

4. \% Difference of "corrected" VAO (ANT) versus "corrected" VAO (RET).

5. Kt/V for both (ANT) and (RET) needle orientation.

6. Recirculation for both (ANT) and (RET) needle orientation.

7. Qb: blood flow ( $\mathrm{mL} / \mathrm{min})$ during UD-Transonic measurements.

According to our findings:

Mean VAO (measured by UD) was lower in the (RET) orientation compared to the (ANT) orientation by $96.21 \mathrm{~mL} / \mathrm{min}(5.66 \%)$.

Using UD, the mean "corrected" VAO for the ANT needle orientation was 1286, $17 \mathrm{~mL} / \mathrm{min}(\mathrm{SD}=455.78,95 \% \mathrm{CI}=1084-1488)$ versus $1189.96 \mathrm{~mL} / \mathrm{min}(\mathrm{SD}=401.05$, $95 \% \mathrm{CI}=1012-1368)$ for the RET orientation. This difference was statistically significant $(p=0.013)$.

The mean $\mathrm{Kt} / \mathrm{V}$ in the (RET) orientation was $1.57(\mathrm{SD}=0.10,95 \% \mathrm{CI}=1.52-1.61)$ versus $1.55(\mathrm{SD}=0.10,95 \% \mathrm{CI}=1.50-1.60)$ for the (ANT) orientation $(p=0.06)$.

Recirculation was $0 \%$ for all measurements.

Mean VAO measured by Doppler was $1079.54 \mathrm{~mL} / \mathrm{min}(\mathrm{SD}=356.04,95 \% \mathrm{CI}=922-1237)$. 


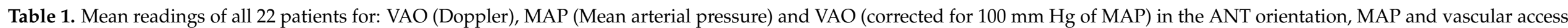

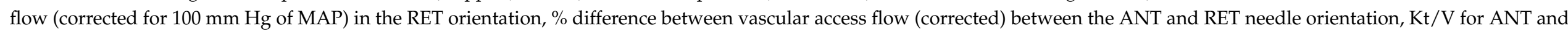
RET orientation, recirculation for both ANT and RET orientation. Qb: blood flow rate during UD-Transonic measurements.

\begin{tabular}{|c|c|c|c|c|c|c|c|c|c|c|}
\hline Patient & $\begin{array}{c}\text { Qb during } \\
\text { Measurements } \\
(\mathrm{mL} / \mathrm{min})\end{array}$ & $\begin{array}{l}\text { VAO Doppler } \\
\text { (Mean) }\end{array}$ & $\begin{array}{c}\text { MAP (Mean) } \\
\text { for VAO } \\
\text { (ANT) }\end{array}$ & $\begin{array}{l}\text { VAO (ANT) } \\
\text { (Mean) } \\
\text { Corrected for } \\
\text { MAP } \\
\text { (100 mm Hg) }\end{array}$ & $\begin{array}{l}\text { MAP (mean) } \\
\text { for VAO } \\
\text { (RET) }\end{array}$ & $\begin{array}{c}\text { VAO (RET) } \\
\text { (Mean) } \\
\text { Corrected for } \\
\text { MAP } \\
\text { (100 mm Hg) }\end{array}$ & $\begin{array}{l}\% \text { Difference of } \\
\text { Corrected VAO } \\
\text { (ANT)-VAO } \\
\text { (RET) }\end{array}$ & Kt/V (ANT) & $\mathrm{Kt} / \mathrm{V}$ (RET) & $\begin{array}{c}\text { Recirculation } \\
\text { ANT/RET }\end{array}$ \\
\hline 1 & 250 & 1450 & 94.67 & 1299.30 & 95.63 & 1149.83 & $11.5 \%$ & 1.48 & 1.45 & $0 \%-0 \%$ \\
\hline 2 & 250 & 900 & 79.65 & 1255.23 & 77.44 & 1236.05 & $1.53 \%$ & 1.6 & 1.65 & $0 \%-0 \%$ \\
\hline 3 & 250 & 1500 & 86.61 & 911.54 & 86.65 & 853.85 & $6.33 \%$ & 1.39 & 1.42 & $0 \%-0 \%$ \\
\hline 4 & 250 & 1000 & 96.63 & 1241.38 & 97.60 & 1197.95 & $3.50 \%$ & 1.41 & 1.56 & $0 \%-0 \%$ \\
\hline 6 & 250 & 1300 & 104 & 1278.85 & 115.33 & 118.50 & $12.54 \%$ & 1.65 & 1.63 & $0 \%-0 \%$ \\
\hline 7 & 250 & 1300 & 86 & 1523.26 & 87 & 1471.26 & $3.41 \%$ & 1.74 & 1.78 & $0 \%-0 \%$ \\
\hline 8 & 250 & 720 & 100 & 600 & 95 & 789.47 & $-31.58 \%$ & 1.55 & 1.54 & $0 \%-0 \%$ \\
\hline 9 & 250 & 850 & 114.67 & 688.95 & 119 & 680.67 & $1.20 \%$ & 1.41 & 1.38 & $0 \%-0 \%$ \\
\hline 10 & 250 & 750 & 96 & 854.17 & 97.33 & 924.66 & $-8.25 \%$ & 1.42 & 1.44 & $0 \%-0 \%$ \\
\hline 11 & 250 & 1700 & 103.33 & 1558.06 & 112.33 & 1513.35 & $2.87 \%$ & 1.55 & 1.6 & $0 \%-0 \%$ \\
\hline 12 & 250 & 750 & 81.32 & 959.02 & 77.34 & 969.83 & $-1.13 \%$ & 1.6 & 1.62 & $0 \%-0 \%$ \\
\hline 13 & 250 & 600 & 104.33 & 651.76 & 104 & 653.85 & $-0.32 \%$ & 1.55 & 1.58 & $0 \%-0 \%$ \\
\hline 14 & 250 & 1800 & 77.28 & 2379.31 & 75.67 & 1982.38 & $16.68 \%$ & 1.54 & 1.51 & $0 \%-0 \%$ \\
\hline 16 & 250 & 800 & 90.65 & 1191.18 & 91.35 & 919.71 & $22.79 \%$ & 1.65 & 1.64 & $0 \%-0 \%$ \\
\hline 17 & 250 & 900 & 79.33 & 1058.82 & 80 & 1000 & $5.56 \%$ & 1.72 & 1.7 & $0 \%-0 \%$ \\
\hline 18 & 250 & 1200 & 58 & 2017.24 & 56 & 2239.14 & $-10.65 \%$ & 1.44 & 1.45 & $0 \%-0 \%$ \\
\hline 19 & 250 & 700 & 96 & 1010.42 & 95.67 & 867.60 & $14.13 \%$ & 1.47 & 1.48 & $0 \%-0 \%$ \\
\hline 20 & 250 & 750 & 75 & 1066.67 & 73 & 958.90 & $10.10 \%$ & 1.52 & 1.54 & $0 \%-0 \%$ \\
\hline 21 & 250 & 900 & 67 & 1640.39 & 65.66 & 1324.87 & $19.23 \%$ & 1.50 & 1.52 & $0 \%-0 \%$ \\
\hline 22 & 250 & 1480 & 91.67 & 1669.09 & 91.33 & 1631.39 & $12.48 \%$ & 1.63 & 1.6 & $0 \%-0 \%$ \\
\hline Mean & 250 & 1079.54 & 88.70 & 1286.17 & 89.11 & 1189.96 & $5.66 \%$ & 1.55 & 1.57 & $0 \%-0 \%$ \\
\hline SD & & 356.04 & 13.46 & 455.78 & 15.93 & 401.05 & $12.48 \%$ & 0.10 & 0.10 & $0 \%-0 \%$ \\
\hline
\end{tabular}


Mean VAO measured by UD-Transonic with (ANT) orientation was $16 \%$ higher than the mean VAO measured by Doppler (1286.17 versus 1079.54, $p=0.009$ ).

Mean VAO measured by UD-Transonic with (RET) orientation was $9.3 \%$ higher than the mean VAO measured by Doppler (1189.96 versus 1079.54, $p=0.113$ ).

We investigated a possible correlation between VA flow (ANT)-VA flow (RET using linear regression analysis. As shown in Figure 1, VA flows using the retrograde and antegrade orientation of the needle correlate well between them $(r=0.93, p<0.001)$.

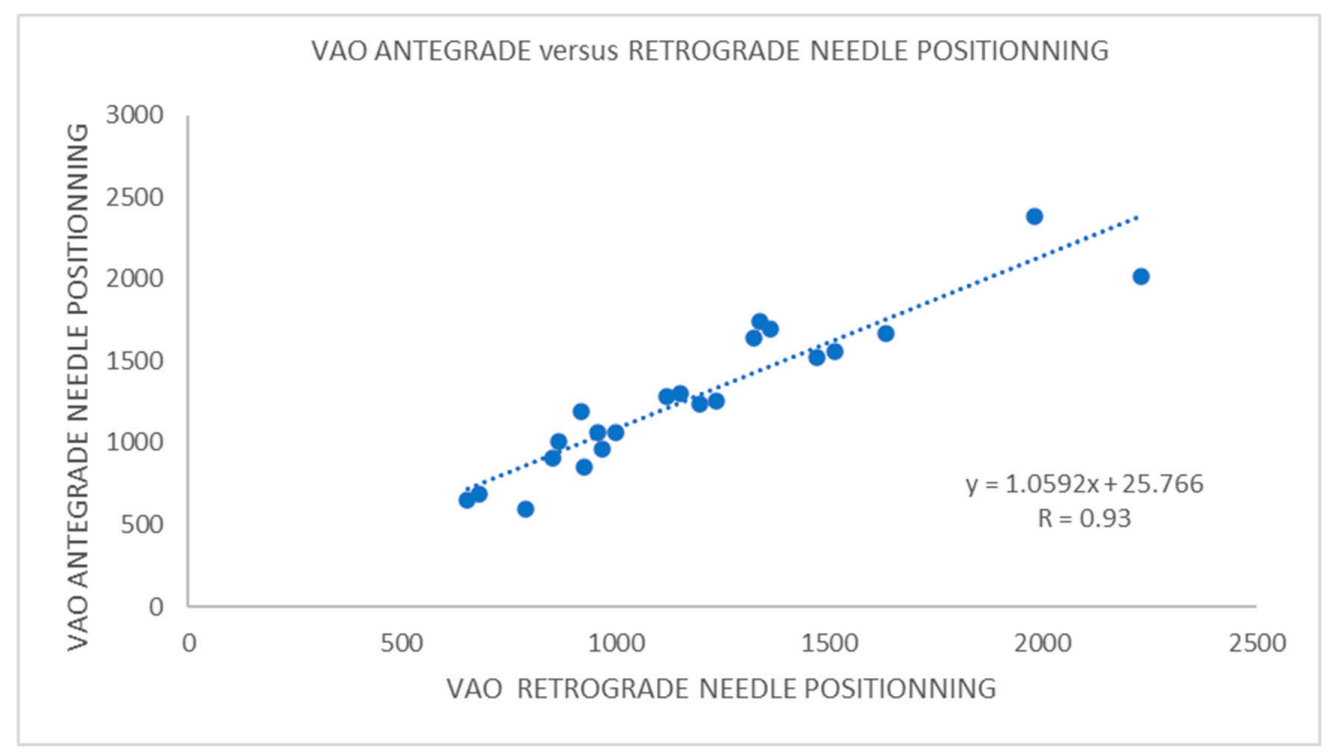

Figure 1. Logistic regression analysis shows a good correlation between VAO measurements with the arterial needle in the antegrade and retrograde orientation $(R=0.93)$.

To test for agreement between the 2 methods (antegrade versus retrograde orientation), we performed a Bland-Altman analysis. The limits of maximum acceptable differences were set to $+/-10 \%$ from bias (Figure 2 ).

\section{Bland Altman plot}

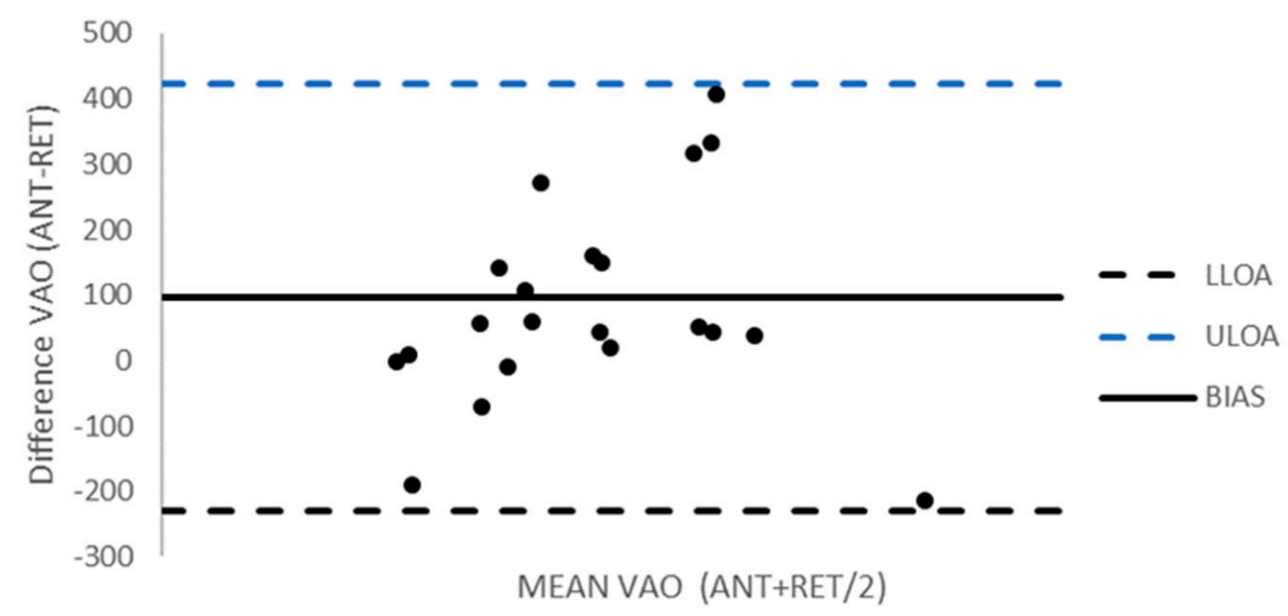

Figure 2. Bland-Altman plot for agreement between VAO measured in the ANT and RET orientation. LLOA: Lower limit of agreement $=-231$ (Bias $-1.96 \times \mathrm{SD})$, ULOA: Upper limit of agreement $=423$ $($ Bias $+1.96 \times \mathrm{SD})$, Bias $=96$. The limits of maximum acceptable differences (not shown) were defined a priori based on clinical criteria: $+/-10 \%$ of bias (79.51-112.89). More than $50 \%$ of results fall outside of these predefined limits (agreement is poor). 


\section{Discussion}

The principal findings of our study, are that the retrograde arterial needle orientation results in statistically significant lower VA flows (by UD) than the antegrade orientation and that VA flows in the RET orientation measured by UD were not statistically significant from VA flows measured by Doppler ultrasound $(p=0.113)$. Moreover, we found that arterial needle orientation did not impact dialysis adequacy or recirculation and that VA flows in the ANT versus RET arterial needle orientation (measured by UD) showed a good correlation but poor agreement.

To our knowledge, our study is the only one that compared VA flows in the RET and ANT arterial needle orientation with VA flows measured by Doppler ultrasound. We believe that the fact that the RET orientation of the arterial needle was not statistically different from the VA flows measured by Doppler ultrasound (contrary to VA flows in the ANT orientation) is an important finding as the Doppler ultrasound is considered a very reliable method for measuring VA flow. Therefore, according to our findings, the RET orientation of the arterial needle should be privileged since it measures more reliably VA flows.

Our study was motivated by Krivitski's original article "Theory and validation of access flow measurement by dilution technique during hemodialysis" (KI 1995) that specifically argues for the retrograde arterial needle orientation to facilitate mixing, an opinion shared by Huisman et al. [10]. Our results confirm Krivitski's opinion and are concordant with the results mentioned by Schoch et al. such that the retrograde arterial needle orientation results in statistically significant lower VA flows than the antegrade orientation. The explanation for these results is not obvious. The UD technique measures access blood flow using the indicator dilution method that is based on Stewart-Hamilton analysis [11,12]. The method consists of injecting a known amount of a known substance $(10 \mathrm{~mL}$ of $0.9 \% \mathrm{NaCl})$ into the venous bloodline (bloodlines are inversed), letting it uniformly mix with blood flow, and then measure its concentration as it passes through a detector (sensor) positioned downstream (arterial line inversed). According to the Stewart-Hamilton equation:

$$
\text { Flow }=\mathrm{m} / \mathrm{Ct} \text {, }
$$

where Flow is measured in $\mathrm{mL} / \mathrm{min}, \mathrm{m}=$ dose of the indicator, $\mathrm{C}=$ concentration, and $\mathrm{t}=$ transit time.

In the abovementioned equation, $\mathrm{m}$ is known, $\mathrm{t}$ is precisely controlled by the UD device, and $C$ is measured by the area below the indicator dilution curve.

Accurate measurement of concentration is very important. The concentration depends on mixing conditions. Mixing is a question of distance and time. As mentioned in the original publication by Krivitski, when the tip of the arterial needle faces the bloodstream (retrograde orientation), mixing is better, takes a little longer, travel distance and travel time increases. This may result in lower concentrations of the indicator in the downstream sensor and a lower VA flow. Therefore, better mixing conditions between the indicator and blood seem to be responsible for the differences observed between access flow measured in the retrograde versus the antegrade arterial needle orientation [13].

To our knowledge, scarce are the studies that have addressed the role of needle orientation on VA flow measurements. Huisman et al. found a certain variability between the ANT and RET needle orientation and suggested that VA flow measurements should be interpreted using the same needle orientation. Schoch et al. [14], in a cohort of 20 patients studied for 6 months found a 9\% higher mean VA flow with the arterial needle in the antegrade orientation compared to the retrograde orientation. Their study didn't compare VA flows with another method.

Our study has several limitations. It is a single-center, not blinded, observational study with no control group with a small study population. Almost $50 \%$ of our patients $(59 / 123)$ did not give informed consent to participate in the study because they feared that the retrograde orientation would potentially harm their access. Although explanations and reassurance were provided, we did not manage to convince them. It was an important 
message and a future challenge for us to try to be more persuasive since longstanding traditions often prove to be an important obstacle for change.

Nevertheless, every effort was made to increase accuracy and precision in our results. All cannulations were realized by the same nurse, proper needle placement was controlled by ultrasound, needle tip distance was the same $(\geq 4 \mathrm{~cm})$ for all measurements. Vessel depth and diameter were measured by ultrasound prior to cannulation [diameter always $>6 \mathrm{~mm}$, (mean $6.4 \mathrm{~mm}$ ) and depth always $<6 \mathrm{~mm}$ (mean $3.2 \mathrm{~mm}$ )]. Moreover, measurements were realized during the first $20 \mathrm{~min}$ of dialysis (period of maximal hemodynamic stability) and flows were "corrected" according to mean arterial pressure in order to eliminate as far as possible a blood pressure effect on flow measurements. Doppler ultrasound VA flows served as a "reference" in order to interpret discrepancies between the VA flows in retrograde and antegrade positions.

It seems that needle distance does not influence the results as long as a 'minimum' distance between needles is respected. In our study, the venous needle tip was always $\geq 4 \mathrm{~cm}$ downstream of the arterial needle tip.

We found important differences in flow between the retrograde and anterograde needle orientation depending on the patients. We do not have a precise explanation for this finding. We took several measures to guarantee the best possible reproducibility of our measurements: cannulation of all AVFs and flow measurements were performed by an experienced staff member (always the same) to respect needle distancing (always $\geq 4 \mathrm{~cm}$ ) and strengthen the internal validity of measurements. Moreover, tip position was verified (before measurements) with ultrasound to avoid needle tip misplacement. One probable (but not proven) explanation is the one proposed by Krivitski (in his original publication) who stated that "when the venous outlet (tip of the arterial needle) does not face the access stream, the situation becomes unpredictable". This "unpredictability" could be the answer to these important differences in flow.

Dialysis adequacy was the same between the RET and the ANT arterial needle orientation. $\mathrm{Kt} / \mathrm{V}$ (1.57 for retrograde versus 1.55 for the antegrade orientation, $p=0.060)$. Our results are in accordance with those of Wiggins et al. [15] and Ozmen et al. that showed that dialysis adequacy was not impacted by the arterial needle orientation.

According to the latest KDOQI and European guidelines [16,17], regular clinical monitoring of VA primes over VA flow surveillance. Nevertheless, as recent research suggests [18] when combined, the two approaches reduce the number of per patient thrombotic events without increasing the number of vascular interventions. Therefore, the accuracy of VA flow measurement is an important issue. Our findings suggest that the use of the retrograde arterial needle orientation should be privileged since it is the more accurate method for VA flow measurement by UD. A multicenter prospective randomized study including a more important number of patients could definitely answer this important question.

Supplementary Materials: The following are available online at https:/ /www.mdpi.com/article/ 10.3390/kidneydial1020017/s1, Figure S1: Photo showing the respective needle orientation used for the study, Figure S2: Photo showing arterial needle positioning (retrograde for the arterial needle-facing bloodstream versus antegrade for the venous needle-not facing the bloodstream).

Author Contributions: G.T.: Concept, data interpretation, preparation of the manuscript; V.L.: Data collection, preparation of the manuscript. All authors have read and agreed to the published version of the manuscript.

Funding: This research received no external funding.

Institutional Review Board Statement: The study was conducted according to the guidelines of the Declaration of Helsinki, and approved by the Institutional Medical Committee (CME-Comité Médicale de l' Etablissement) of the CENTRE DE NEPHROLOGIE DE MONTARGIS-Approval number 1-12032021, date of approval 12 March 2021).

Informed Consent Statement: Informed consent was obtained from all subjects involved in the study.

Data Availability Statement: Not applicable. 
Conflicts of Interest: The authors declare no conflict of interest.

\section{References}

1. Haas, G. Ueber Versuche der Blutauswaschung am Lebenden mit Hilfe der Dialyse. Archiv. Exp. Path. Pharmakol. 1926, 106, 158-172. [CrossRef]

2. Brescia, M.J.; Cimino, J.E.; Appel, L.; Hurwich, B.J. Chronic hemodialusis using venipuncture and a surgically created arteriovenous fistula. N. Engl. J. Med. 1966, 275, 1089-1092. [CrossRef] [PubMed]

3. Parisotto, M.T.; Schoder, V.U.; Miriunis, C.; Grassmann, A.H.; Scatizzi, L.P.; Kaufmann, P.; Stopper, A.; Marcelli, D. Cannulation technique influences arteriovenous fistula and graft survival. Kidney Int. 2014, 86, 790-797. [CrossRef] [PubMed]

4. Gauly, A.; Parisotto, M.T.; Skinder, A.; Scoder, V.; Furlan, A.; Schuh, E.; Marcelli, D. Vascular access cannulation in hemodialysis patients-a survey of current practice and its relation to dialysis dose. J. Vasc. Access 2011, 12, 358-364. [CrossRef] [PubMed]

5. Reyes, O.R., II. Effects of arterial needle placement in arteriovenous fistula on dialysis adequacy of end-stage renal disease patients undergoing maintenance hemodialysis. J. Nurs. Healthc. 2016, 1, 1-10.

6. Ozmen, S.; Kadiroglu, A.K.; Ozmen, C.A.; Danis, R.; Sit, D.; Akin, D.; Yilmaz, M.E. Does the direction of arterial needle in Av fistula cannulation affect dialysis adequacy? Clin. Nephrol. 2008, 70, 229-232. [CrossRef]

7. Fulker, D.; Simmons, A.; Barber, T. Computational model of the arterial and venous needle during hemodialysis. J. Biochem. Eng. 2017, 139, 11005. [CrossRef] [PubMed]

8. Kopple, J.D. National Kidney Foundation: K/DOQI Clinical Practice Guidelines for Vascular Access: AJKD. Am. J. Kidney Dis. 2020, 75, S1-S64.

9. Krivitski, N.M. Theory and validation of access flow measurement by dilution technique during hemodialysis. Kidney Int. 1995, 48, 244-250. [CrossRef] [PubMed]

10. Huisman, R.; van Dijk, M.; de Bruin, C.; Loonstra, J.; Sluiter, W.; Zeebregts, C.; van der Dungen, J. Within-session and between session variability of hemodialysis shunt flow measurements. Nephrol. Dial. Transplant. 2005, 12, 2842-2847. [CrossRef] [PubMed]

11. Stewart, G.N. Researches on the circulation time and on the influences which affect it. J. Physiol. 1897, 22, 159. [CrossRef] [PubMed]

12. Hamilton, W.F. Assessment of the Fick and dye injection methods of measuring cardiac output in man. Am. J. Physiol. Leg. Content 1948, 153, 309-321. [CrossRef] [PubMed]

13. Depner, T.; Krivitski, N. Clinical measurement of blood flow in hemodialysis access fistulae and grafts by ultrasound dilution. $J$. Am. Soc. Artif. Intern. Organs 1995, 41, 745-749. [CrossRef] [PubMed]

14. Schoch, M.; Wilson, S.; Agar, J.W.M. Variations in vascular access flow in hemodialysis can depend on arterial needle orientation. Ren. Soc. Aust. J. 2008, 4, 45-50.

15. Wiggins, K.; Agar, J.W.M.; Somerville, C.A. A controlled study of arterial needle direction on dialysis adequacy in patients undergoing hemodialysis through a radiocephalic arteriovenous fistula. Nephrology 2003, 8, 70.

16. Lok, C.E.; Huber, T.S.; Lee, T.; Shenoy, S.; Yevzlin, A.S.; Abreo, K.; Allon, M.; Asif, A.; Astor, B.C.; Glickman, M.H.; et al. KDOQI Clinical Practice Guideline for Vascular Access 2019 Update. Am. J. Kidney Dis. 2020, 75, S1-S164. [CrossRef] [PubMed]

17. Schmidli, J.; Widmer, M.K.; Basile, C.; de Donato, G.; Gallieni, M.; Gibbons, C.P.; Haage, P.; Hamilton, G.; Hedin, U.; Kamper, L.; et al. 2018 Clinical Practice Guidelines of the European Society of Vascular Surgery (ESVS). Eur. J. Vasc. Endovasc. Surg. 2018, 55, 757-818. [CrossRef] [PubMed]

18. Salman, L.; Rizvi, A.; Contreras, G.; Manning, C.; Feustel, P.J.; Machado, I.; Briones, P.L.; Jamal, A.; Bateman, N.; Martinez, L.; et al. A Multicenter Randomized Clinical Trial of Hemodialysis Access Blood Flow Surveillance Compared to Standard of Care: The Hemodialysis Access Surveillance Evaluation (HASE) Study. Kidney Int. Rep. 2020, 5, 1937-1944. [CrossRef] [PubMed] 